\title{
Tzitzeica-Type centro-affine invariants in Minkowski spaces
}

\author{
Alexandru Bobe, Wladimir G. Boskoff and Marian G. Ciucă
}

\begin{abstract}
In this article we introduce three centro-affine invariant functions in Minkowski spaces. Tzitzeica curves and Tzitzeica surfaces may be defined in this new context. Our main results allow us to understand the connections between Tzitzeica curves and surfaces in Minkowski spaces and the original Tzitzeica curves and surfaces from the Euclidian space.
\end{abstract}

\section{Introduction}

Gheorghe Tzitzeica provided one of the first major contributions to affine differential geometry in the early twentieth century when he published his results on "S-surfaces", later to be known as Tzitzeica surfaces [9, 10]. Indeed, these works may be considered to be the starting point of the affine differential geometry as it can be seen in [4] and [6] (for further information on the pioneering work of Titeica, see [1]).

In the sequel, we review this pioneering work of Tzitzeica, by introducing the notion of Tzitzeica function for a curve and for a surface. All the results are presented in a 3-dimensional Minkowski space.

Key Words: Tzitzeica curve, Tzitzeica surface, centro-affine invariants, Minkowski space.

2010 Mathematics Subject Classification: 51B20, 53A04, 53A05, 53A15.

Received: August, 2011.

Accepted: February, 2012. 


\section{The Tzitzeica function for curves in $\mathbb{R}_{1}^{3}$}

The space $\mathbb{R}_{1}^{3}$ is defined as a space to be the usual three-dimensional $\mathbb{R}$-vector space consisting of vectors $\left\{\left(x_{1}, x_{2}, x_{3}\right) \mid x_{1}, x_{2}, x_{3} \in \mathbb{R}\right\}$, but endowed with the inner product

$$
\langle X, Y\rangle_{1}=-x_{1} y_{1}+x_{2} y_{2}+x_{3} y_{3} .
$$

This space is called the 3 -dimensional Minkowski space ([5]). According to the definitions from [5] the Minkowski norm is defined as $\|X\|_{1}=\sqrt{\left|\langle X, X\rangle_{1}\right|}$ and two vectors $X$ and $Y$ are Minkowski orthogonal if $\langle X, Y\rangle_{1}=0$.

More, consider $X=\left(x_{1}, x_{2}, x_{3}\right)=x_{1} \vec{i}+x_{2} \vec{j}+x_{3} \vec{k}$ and $Y=\left(y_{1}, y_{2}, y_{3}\right)=$ $y_{1} \vec{i}+y_{2} \vec{j}+y_{3} \vec{k}$. The Minkowski vectorial product $X \times_{1} Y$ is defined by the formula

$$
X \times_{1} Y:=\left|\begin{array}{ccc}
-\vec{i} & \vec{j} & \vec{k} \\
x_{1} & x_{2} & x_{3} \\
y_{1} & y_{2} & y_{3}
\end{array}\right|
$$

Let $c: I \subset \mathbb{R} \longrightarrow \mathbb{R}_{1}^{3}$ be a regular curve in the 3-dimensional Minkowski space (that is $\|\dot{c}(t)\|_{1} \neq 0$ ). Denote the torsion of the curve $c$ at the point $c(t)$ by $K_{2}(c)(t)$, and denote the distance from the origin to the osculating plane at the point $c(t)$ by $d_{c}(t)$.

Lemma 2.1. Let $c: I \subset \mathbb{R} \longrightarrow \mathbb{R}_{1}^{3}, c(t)=(x(t), y(t), z(t))$ be a regular curve in the 3-dimensional Minkowski space. Then we have the relation:

$$
K_{2}(c)(t)=d_{c}^{2}(t) \cdot I_{c}(t)
$$

where $I_{c}(t)=\frac{\operatorname{det}(\dot{c}(t), \ddot{c}(t), \ddot{c}(t))}{(\operatorname{det}(c(t), \dot{c}(t), \ddot{c}(t)))^{2}}$ is a function of the variable $t$.

Proof. We'll first find the torsion of the curve in terms of the quantities which appear in the analytic equations of the Frenet frame. According to [5] the torsion of the curve is

$$
K_{2}(c)(t)=\frac{\operatorname{det}(\dot{c}(t), \ddot{c}(t), \ddot{c}(t))}{\left\|\dot{c}(t) \times_{1} \ddot{c}(t)\right\|_{1}^{2}}
$$

On the other hand, the osculating plane at the point $c(t)$ is the plane spanned by $\dot{c}(t)$ and $\ddot{c}(t)$. Thus, $d_{c}(t)$ is the projection of $c(t)$ onto the normal direction of this plane, that is

$$
d_{c}(t)=\left\langle c(t), \frac{\dot{c}(t) \times_{1} \ddot{c}(t)}{\left\|\dot{c}(t) \times_{1} \ddot{c}(t)\right\|_{1}}\right\rangle_{1}=\frac{\left\langle c(t), \dot{c}(t) \times_{1} \ddot{c}(t)\right\rangle_{1}}{\left\|\dot{c}(t) \times_{1} \ddot{c}(t)\right\|_{1}} .
$$


Putting this together with the expression for torsion, we have

$$
\frac{K_{2}(c)(t)}{d_{c}^{2}(t)}=\frac{\operatorname{det}(\dot{c}(t), \ddot{c}(t), \ddot{c}(t))}{(\operatorname{det}(c(t), \dot{c}(t), \ddot{c}(t)))^{2}}=I_{c}(t)
$$

Such kind of function appears in [7] and [8] for curves in Euclidian spaces but expressed in terms of differential forms. A coordinate computation leads to the formula above. In [7] and [8] is proved that the previous function is a centro-affine invariant. Is this possible in our Minkowski context?

Definition 2.2. We call the function $I_{c}$ from lemma 2.1 the Tzitzeica function for the curve $c: I \subset \mathbb{R} \longrightarrow \mathbb{R}_{1}^{3}$ in the Minkowski space $\mathbb{R}_{1}^{3}$.

Definition 2.3. A centro-affine transformation of the curve $c: I \subset$ $\mathbb{R} \longrightarrow \mathbb{R}_{1}^{3}, c(t)=(x(t), y(t), z(t))$ is a curve $h: I \subset \mathbb{R} \longrightarrow \mathbb{R}_{1}^{3}, h(t)=c(t) \cdot M$, where $M \in M_{3}(\mathbb{R})$, $\operatorname{det} M \neq 0$.

Definition 2.4. Let $f_{c}: I \subset \mathbb{R} \longrightarrow \mathbb{R}$ be a function associated to a curve $c . f_{c}$ is called an centro-affine invariant for the curve $c$ if, for any centro-affine transformation $h$, it exists $k \in \mathbb{R}$ such that $f_{h}(t)=k \cdot f_{c}(t), \forall t \in I \subset \mathbb{R}$.

In the following we prove that the Tzitzeica function for a curve in the Minkowski space $\mathbb{R}_{1}^{3}$ is a centro-affine invariant for the given curve.

Theorem 2.5. Let $h: I \subset \mathbb{R} \longrightarrow \mathbb{R}_{1}^{3}$ be a centro-affine transformation of a curve $c: I \subset \mathbb{R} \longrightarrow \mathbb{R}_{1}^{3}, c(t)=(x(t), y(t), z(t))$. Then the Tzitzeica function $I_{c}$ is a centro-affine invariant of the curve $c$, and furthermore it satisfies the relation

$$
I_{h}(t)=\frac{1}{\operatorname{det} M} \cdot I_{c}(t)
$$

Proof: Let $M \in M_{3}(\mathbb{R})$ be a nonsingular matrix. Then the corresponding centro-affine transformation of the curve $c$ has the form:

$$
h(t)=c(t) \cdot M=\left(x_{1}(t), y_{1}(t), z_{1}(t)\right) .
$$

The following equalities can be deduced after some computations,

$$
\begin{aligned}
& \operatorname{det}(\dot{h}(t), \ddot{h}(t), \ddot{h}(t))=\operatorname{det} M \cdot \operatorname{det}(\dot{c}(t), \ddot{c}(t), \ddot{c}(t)), \\
& \operatorname{det}(h(t), \dot{h}(t), \ddot{h}(t))=\operatorname{det} M \cdot \operatorname{det}(\dot{c}(t), \ddot{c}(t), \ddot{c}(t)) .
\end{aligned}
$$


Using the relationship between the torsion and the distance $d_{c}(t)$ found in lemma 2.1, we can deduce the relation which the centro-affine transformation $h(t)$ satisfies: $I_{h}(t)=\frac{K_{2}(h)(t)}{d_{h}^{2}(t)}=\frac{\operatorname{det}(\dot{h}(t), \ddot{h}(t), \dddot{h}(t))}{(\operatorname{det}(h(t), \dot{h}(t), \ddot{h}(t)))^{2}}=\frac{\operatorname{det} M}{(\operatorname{det} M)^{2}}$. $\frac{\operatorname{det}(\dot{c}(t), \ddot{c}(t), \dddot{c}(t))}{(\operatorname{det}(c(t), \dot{c}(t), \ddot{c}(t)))^{2}}=\frac{1}{\operatorname{det} M} \cdot I_{c}(t)$

Using the above result, we can obtain as a corollary the equivalent of the theorem of Tzitzeica concerning curves in a Minkowski space.

Definition 2.6. A curve $c: I \subset \mathbb{R} \longrightarrow \mathbb{R}_{1}^{3}$ is called a Tzitzeica curve in the 3-dimensional Minkowski space if the Tzitzeica function $I_{c}$ is constant (Tzitzeica condition).

Corollary 2.7. The centro-affine transformation of a Tzitzeica curve is a Tzitzeica curve. The relation between the constants is

$$
I_{h}=\frac{1}{\operatorname{det} M} \cdot I_{c} .
$$

Proof: We know from hypothesis that $c$ is a Tzitzeica curve, so $\frac{K_{2}(c)(t)}{d_{c}^{2}(t)}=$ $I_{c} \in \mathbb{R}$. Using theorem 2.5 we obtain that $\frac{K_{2}(h)(t)}{d_{h}^{2}(t)}=I_{h} \in \mathbb{R}$, as needed.

Let us observe that a centro-affine transformation in a 3-Minkowski space may change the type of the curve $c$, that is, if the speed vector initialy is a temporal one, after such a transformation it can be a spatial one. Nevertheless, the torsion in a point of the new curve $h$ and the distance from the origin to the osculating plane at the corresponding point can be computed and the ratio $\frac{K_{2}(t)(t)}{d_{h}^{2}(t)}$ makes sense. So, we can identify the curves $c$ for which the ratio $\frac{K_{2}(c)(t)}{d_{c}^{2}(t)}$ is a constant and according to our Corrolary 2.7 the constancy is preserved for any centro-affine transformation. In fact, if the differential equation $I_{c}(t)=$ const. is satisfied for a curve $c$, it is satisfied for any $h$, $h(t)=c(t) \cdot M$. In [7] the same type differential equation is satisfied by Tzitzeica curves in an Euclidian space. That is, there is a same solution $c$ in both cases. So, even if the geometric meaning of the curves may be different, we can say that the curves from Euclidian space which satisfies the Tzitzeica condition are curves which satisfies the Tzitzeica condition in Minkowski space and viceversa. As an example, we can choose a Minkowski circle which is a Tzitzeica curve in the Minkowski space; its equivalent in the Euclidian space it is a equilateral hyperbola which is also a Tzitzeica curve, but in the Euclidian space. Some other examples of Tzitzeica curves can be seen in [3]. The geometric meaning of these objects depends on the geometry but they both fullfiled the Tzitzeica condition. 


\section{The Tzitzeica function for surfaces in $\mathbb{R}_{1}^{3}$}

Let $f: U=\stackrel{\circ}{U} \subset \mathbb{R}^{2} \longrightarrow \mathbb{R}_{1}^{3}$ be a surface in the Minkowski space $\mathbb{R}_{1}^{3}$ given by $f(x, y)=(x, y, u(x, y))$. We denote by $K_{f}(p)$ the Gauss curvature of $f$ at the point $p=(x, y)$ and by $d_{f}(p)$ the distance from the origin to the tangent plane of the surface $f$ at the point $f(p)$. According to [5] the formula for the Gausssian curvature is $K_{f}(p)=\frac{\frac{\partial^{2} u}{\partial x^{2}} \cdot \frac{\partial^{2} u}{\partial y^{2}}-\left(\frac{\partial^{2} u}{\partial x y y}\right)^{2}}{\epsilon\left|-\left(\frac{\partial u}{\partial x}\right)^{2}+\left(\frac{\partial u}{\partial y}\right)^{2}+1\right|^{2}}$, where $\epsilon$ is a sign defined by the Gauss map associated to $f$.

Lemma 3.1. The surface satisfies:

$$
K_{f}(p)=d_{f}^{4}(p) \cdot J_{f}(p),
$$

where

$$
J_{f}(p)=\epsilon \frac{\frac{\partial^{2} u}{\partial x^{2}} \cdot \frac{\partial^{2} u}{\partial y^{2}}-\left(\frac{\partial^{2} u}{\partial x \partial y}\right)^{2}}{\left|u(x, y)-x \frac{\partial u}{\partial x}-y \frac{\partial u}{\partial y}\right|^{4}} .
$$

Proof: If we compute $\frac{\partial f}{\partial x} \times{ }_{1} \frac{\partial f}{\partial y}$ we obtain the vector $\left|\begin{array}{ccc}-\vec{i} & \vec{j} & \vec{k} \\ 1 & 0 & \frac{\partial u}{\partial x} \\ 0 & 1 & \frac{\partial u}{\partial y}\end{array}\right|$, that is $\frac{\partial f}{\partial x} \times_{1} \frac{\partial f}{\partial y}=\left(\frac{\partial f}{\partial x},-\frac{\partial f}{\partial y}, 1\right)$.

Then the equation of the tangent plane in a given point $(x, y, u(x, y))$ is $-\frac{\partial u}{\partial x}(X-x)+\left(-\frac{\partial u}{\partial y}\right)(Y-y)+(Z-u(x, y))=0$.

It means that the distance from the origin to the tangent plane of the surface $f$ in the point $f(p)$ is:

$$
d_{f}(p)=\frac{\left|x \frac{\partial u}{\partial x}+y \frac{\partial u}{\partial y}-u(x, y)\right|}{\sqrt{\left|-\left(\frac{\partial u}{\partial x}\right)^{2}+\left(\frac{\partial u}{\partial y}\right)^{2}+1\right|}} .
$$

If we compute $\frac{K_{f}(p)}{d_{f}^{4}(p)}$ we obtain $\epsilon \frac{\frac{\partial^{2} u}{\partial x^{2}} \cdot \frac{\partial^{2} u}{\partial y^{2}}-\left(\frac{\partial^{2} u}{\partial x \partial y}\right)^{2}}{\left|u(x, y)-x \frac{\partial u}{\partial x}-y \frac{\partial u}{\partial y}\right|^{4}}$ that is the $J_{f}(p)$ from the enounce.

Definition 3.2. We call the function $J_{f}$ from lemma 3.1 the Tzitzeica function for the surface $f$ in the Minkowski space $\mathbb{R}_{1}^{3}$. 
Definition 3.3. A centro-affine transformation of a surface $f$ is a surface $g: U=\stackrel{\circ}{U} \subset \mathbb{R}^{2} \longrightarrow \mathbb{R}_{1}^{3}$ given by

$$
g(x, y)=f(x, y) \cdot M,
$$

where $M \in M_{3}(\mathbb{R})$, det $M \neq 0$.

Definition 3.4. Let $A_{f}: U \subset \mathbb{R}^{2} \longrightarrow \mathbb{R}$ be a function associated to a surface $f . A_{f}$ is called an centro-affine invariant for the surface $f$ if, for any centro-affine transformation $g$, it exists $k \in \mathbb{R}$ such that $A_{g}(p)=k \cdot A_{f}(p)$, $\forall p \in U \subset \mathbb{R}^{2}$.

In the following we prove that the Tzitzeica function for a surface in the Minkowski space $\mathbb{R}_{1}^{3}$ is an centro-affine invariant for the given surface.

Theorem 3.5. Let $f: U=\stackrel{\circ}{U} \subset \mathbb{R}^{2} \longrightarrow \mathbb{R}_{1}^{3}$ be a surface and $g$ a centroaffine transformation of the surface $f$. Then the Tzitzeica function $J_{f}$ is a centro-affine invariant, and furthermore it satisfies the relation:

$$
J_{g}(p)=\frac{-1}{(\operatorname{det} M)^{2}} \cdot J_{f}(p) .
$$

Proof: Let us consider $g$ a centro-affine transformation of a surface $f$ given by $g(x, y)=f(x, y) \cdot M$, where $M \in M_{3}(\mathbb{R})$, $\operatorname{det} M \neq 0$.

The following equalities can be proved after computations

$$
K_{g}=(-\operatorname{det} M)^{2} \cdot K_{f}
$$

and $d_{g}=|\operatorname{det} M| \cdot d_{f}$.

It rezults

$$
J_{g}=\frac{K_{g}}{d_{g}^{4}}=\frac{-1}{(\operatorname{det} M)^{2}} \cdot J_{f} . \square
$$

Using the above theorem, we can obtain as a corollary the theorem of Tzitzeica concerning surfaces.

Definition 3.6. A surface $f: U=\stackrel{\circ}{U} \subset \mathbb{R}^{2} \longrightarrow \mathbb{R}_{1}^{3}$ is called Tzitzeica surface in the Minkowski space $\mathbb{R}_{1}^{3}$ if the Tzitzeica function $J_{f}$ is constant.

Corollary 3.7. In a Minkowski 3-dimensional space a centro-affine transformation of a Tzitzeica surface is a Tzitzeica surface. Furthermore the centroaffine invariant satisfies the relation $J_{g}=\frac{-1}{(\operatorname{det} M)^{2}} \cdot J_{f}$. 
Proof: Knowing that $f$ is a Tzitzeica surface we have that $J_{f}$ is constant, and using theorem 3.5 we obtain immediatly the conclusion and the relation. $\square$

Let us see the connection with the Tzitzeica surfaces in the Euclidian space. In [7] and [8] we can see an equivalent of the Tzitzeica invariant function. In coordinates, if we compute $\frac{K_{g}}{d_{g}^{4}}$ we obtain the affine invariant $\frac{\frac{\partial^{2} u}{\partial x^{2}} \cdot \frac{\partial^{2} u}{\partial y^{2}}-\left(\frac{\partial^{2} u}{\partial x \partial y}\right)^{2}}{\left(u(x, y)-x \frac{\partial u}{\partial x}-y \frac{\partial u}{\partial y}\right)^{4}}$. That is the equivalent equation of the Tzitzeica surfaces in the Euclidian space is $\frac{\partial^{2} u}{\partial x^{2}} \cdot \frac{\partial^{2} u}{\partial y^{2}}-\left(\frac{\partial^{2} u}{\partial x \partial y}\right)^{2}=k\left(u(x, y)-x \frac{\partial u}{\partial x}-y \frac{\partial u}{\partial y}\right)^{4}$. This formula is up to a sign the function of Tzitzeica surfaces in Minkowski spaces. As in the case of Tzitzeica curves, we can assert that objects which are Tzitzeica surfaces in the Euclidian space are Tzitzeica surfaces in the Minkowski space, too, even if the geometric meaning of the objects is different. The inverse is also true. As an example let us consider the Minkowski sphere $-x^{2}+y^{2}+z^{2}=1$. It is easy to see that this surface is a Tzitzeica surface in the Minkowski 3-dimensional space $\mathbb{R}_{1}^{3}$, but the equation of this Minkowski sphere in the Euclidian 3-dimensional space represents an hyperboloid which is different to the Euclidian sphere. The Tzitzeica surface induced by the equation of the Euler's line of a triangle as in [2] is an example of Tzitzeica surface in a Minkowski space according to our theory, too. Then the equations of Tzitzeica surfaces in Minkowski spaces are equations of Tzitzeica surfaces in Euclidian spaces and viceversa.

\section{References}

[1] A. Agnew, A. Bobe, W. Boskoff and B. Suceava, Gheorghe Ţiţeica and the origins of affine differential geometry. Hist. Math. 36 (2009), 161-170.

[2] A. Agnew, A. Bobe, W. Boskoff, L. Homentcovschi and B. Suceava, The equation of Euler's line yields a Tzitzeica surface. Elem. Math. 64 (2009), 71-80.

[3] A. Agnew, A. Bobe, W. Boskoff and B. Suceava, Tzitzeica Curves and Surfaces. The Math. Journal 12 (2010), 1-18.

[4] S. Buchin, Affine Differential Geometry. Science Press, Beijing, China, and Gordon and Breach, Science Publishers, Inc., New York, 1983

[5] W. Kühnel, Differential Geometry. Curves-Surfaces-Manifolds, 2nd Ed. AMS, 2000.

[6] K. Nomizu and T. Sasaki, Affine Differential Geometry. Cambridge University Press, 1995. 
[7] M. Putinar, Sur les courbes et hypersurfaces de Tzitzéica. Bull. Math. Soc. Sci. Math. R. S. Roumanie, 23(71)(1979), no. 4, 419-426.

[8] M. Putinar, Un invariant centro-affine associé à quelques sous-variétés de l'espace euclidien. Rev. Roumaine Math. Pures Appl. 24 (1979), no. 4, 647-649.

[9] G. Titeica, Sur une nouvelle classe de surfaces. C.R. Acad. Sci., Paris, Ser. I, Math. 144 (1907), 1257-1259.

[10] G. Tzitzeica, Sur une nouvelle classe de surfaces. Rendiconti del Circolo Matematico di Palermo 25 (1908), 180-187.

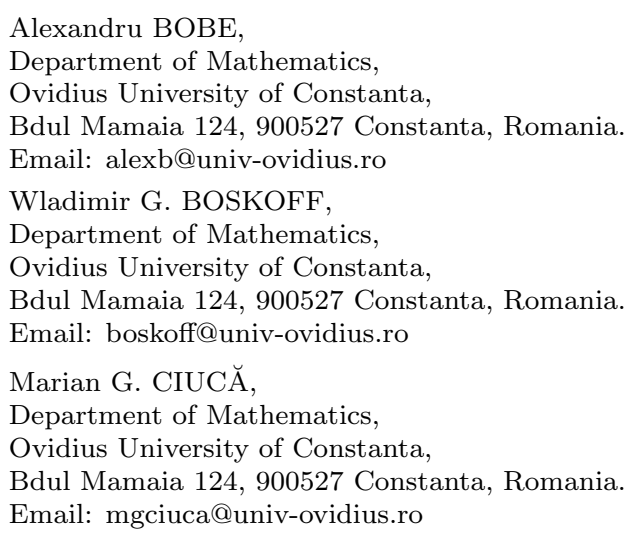

